\title{
Estudio citomorfológico de la maduración de gónadas de hembras de Seriola lalandi en un Sistema Acuícola de Recirculación (SAR)
}

\author{
Cytomorphological study about maturation of female gonads \\ of Seriola lalandi from a Recirculating Aquaculture System (RAS) \\ Hipólito Núñez ${ }^{1}$ Bernardo Arriaza ${ }^{3}$, Nicolás Garrido ${ }^{1,2}$ y Arnaldo Vilaxa ${ }^{1,2 *}$
}

\section{RESUMEN}

Seriola lalandi es un pez que tiene descrita su biología reproductiva en especímenes silvestres. En particular, la maduración gonadal de este pez presenta variaciones según el sexo y largo de cada individuo. No obstante, en peces en cautiverio, como es el caso de su cultivo en un Sistema Acuícola de Recirculación (SAR), para su engorda y posterior comercialización, aún no se ha descrito su desarrollo gonadal ni su asociación con el largo o peso del pez. Para contribuir a este vacío se seleccionaron, durante un año, 30 individuos provenientes del Centro de Estudios Marinos y Limnológicos (CEMYL) de la Universidad de Tarapacá. A estos se les realizó la eutanasia para posteriormente realizarles la necropsia correspondiente, extrayendo las gónadas, a las que se le aplicaron técnicas histológicas de rutina (Hematoxilina - Eosina) y técnicas histoquímicas (Picro - Fucsina de Van Gieson y Periodic Acid Schiff). Obteniendo como resultado la identificación de seis hembras, de las que cinco estaban en una etapa inmadura de crecimiento gonadal presentando solo oocitos inmaduros en los cuales el núcleo y nucléolo eran más abundantes que el citoplasma y una de ellas presentaba solo oogonios que son los precursores de los oocitos. Se concluye que no se puede determinar la maduración gonadal en individuos con tallas menores a $40 \mathrm{~cm}$, como los analizados en este estudio.

Palabras clave: teleósteo, cavidad celómica, peritoneo mesovárico, maduración gonadal.

\begin{abstract}
Seriola lalandi is a fish that has described their reproductive biology in wild specimens. In particular, the gonadal maturation of this fish has variations by sex and length of each individual. However, fish in captivity, as in the case of cultivation in Aquaculture Recirculation System (SAR), for fattening and subsequent marketing, has not yet been described their gonadal development and its association with long or weight of fish. To contribute to this gap, were selected for a year, 30 individuals from the Center for Marine Studies and Limnological (CEMYL) at the University of Tarapaca. These were euthanized in order to conduct the corresponding autopsy, removing the gonads, to which were applied Histological Techniques routine (Hematoxylin - Eosin) and Histochemical Techniques (Picro - Fuchsin Van Gieson and Periodic Acid Schiff). Which resulted in the identification of 6 female, 5 of which were in an immature stage of gonadal growth presenting only oocytes immature in which the nucleus and nucleolus were more abundant than the cytoplasm and one of them had only oogonia that are the precursors oocytes. It is concluded that you can not determine the gonadal maturation in individuals with smaller sizes to $40 \mathrm{~cm}$, as analyzed in this study.
\end{abstract}

Key words: teleost, coelomic cavity, mesovárico peritoneum, gonadal maturation.

\section{Introducción}

La especie Seriola lalandi, pez pelágico de la familia Carangidae, conocido como vidriola, dorado o palometa (Ruiz, 2012). Esta especie se presenta en la acuicultura mundial con muy buenos resultados en ámbitos de la economía por poseer un crecimiento rápido, es así que en 18 meses puede alcanzar un peso de 3-4 $\mathrm{kg}$ y su precio en el mercado puede fluctuar entre US\$7-8 $/ \mathrm{kg}$ (fresco refrigerado) y altos niveles de omega tres, esto está respaldado por el Programa de Diversificación Acuícola de Chile (PDACh) que visualizan a ser una especie con un alto potencial acuícola a muy corto plazo en Chile (Silva, 2011).

Las características reproductivas de Seriola lalandi han sido documentadas a partir de peces

\footnotetext{
Departamento de Biología, Universidad de Tarapacá, Arica, Chile. Centro de Estudios Marinos y Limnológicos (CEMYL - UTA).

Instituto de Alta Investigación, Universidad de Tarapacá, Arica, Chile.

* Autor por correspondencia: avilaxa@uta.cl
}

Fecha de Recepción: 9 Agosto, 2016.

Fecha de Aceptación: 22 Septiembre, 2016.

DOI: $10.4067 / S 0718-34292016005000037$. 
recolectados desde 1974 hasta 2012, en Nueva Zelandia y en otros países del Asia Pacífico, estos peces son de vida libre, y en ellos se logró determinar que la hembras maduran a $83,4 \mathrm{~cm}$ de largo y los machos a 47,1 cm de largo (Fielder \& Heasman, 2011). Shiraishi et al., (2010), lograron establecer que Seriola lalandi, alcanza su periodo de maduración sexual para ambos sexos a los 2 años.

Poortenaar et al. (2001), indicó criterios para el análisis y clasificación de las gónadas de hembras de Seriola lalandi, estos criterios se basaron en las características macroscópicas e histológicas.

En nuestro país en la última década se ha estado realizando el cultivo y estudio de su comportamiento en cautiverio, es así, que los primeros informes de Aguilera et al. (2013), están relacionados con el estudio de la flora intestinal. En el 2014 se presenta un estudios sobre el desarrollo morfológico y el crecimiento alométrico de larvas de Seriola lalandi en condiciones de cultivo (Martínez-Montaño et al. 2014).

Es por esto que lograr determinar y asociar los estadios de maduración gonadales de Seriola lalandi en cautiverio, cobran gran importancia, ya que los peces en esta condición tienen un comportamiento y fisiología diferente. Los datos obtenidos, hasta la fecha de este pez, se relaciona a investigaciones realizadas en especímenes silvestres en el continente de Oceanía (Ottolenghi et al., 2004). La información obtenida de los distintos estadios gonadales de esta especie en cautiverio, generará datos importantes para pensar en un cultivo intensivo de esta especie en el continente americano, en especial en la zona norte de nuestro país.

\section{Material y Método}

En esta investigación se utilizaron 30 peces aportados por el Centro de Estudios Marinos y Limnológicos (CEMYL), de la Universidad de Tarapacá. A estos peces se les realizó la eutanasia y luego la necropsia correspondiente, a las gónadas extraídas del pez y de distintas edades fueron fijadas en Formalina Tamponada al 10\%, cada una de ellas fue procesada aplicando las técnicas histológicas de rutina, se realizarón cortes de $5 \mu \mathrm{m}$ y se tiñieron con Hematoxilina - Eosina y técnicas histoquímicas de Picro - Fucsina de Van Gieson y P.A.S.

Para el análisis citomorfológico del tejido gonadal, de Seriola lalandi se utilizó un microscopio de marca Olympus modelo CX-41 con cámara integrada utilizando el software Micrometrics $S E$ Premium

\section{Resultados y Discusión}

Los cortes histológicos de las seis gónadas de hembras encontradas en los especímenes de Seriola lalandi, se les pudo describir un delgado tejido externo a la gónada, que corresponde a un tejido conjuntivo denso regular o modelado, constituido por fibras colágenas ordenadas, además presenta un epitelio plano simple que la recubre completamente (mesotelio), estos dos constituyentes dan origen a la albugínea ovárica. El mismo tejido conjuntivo, pero más delgado se adentra a la zona medular de la gónada para formar los tabiques de los lóbulos con características asimétricas (unos más gruesos que otros), en cuyo interior se encuentran y maduran las células germinales llamadas oocitos, estas son células de reducido tamaño (entre 20 a $50 \mu \mathrm{m}$ ), y según su grado de maduración, pueden ser redondas u ovoide con un núcleo vesiculoso y de posición central (Figura 1). El corte histológico de la gónada correspondiente a la muestra $\mathrm{N}^{\circ} 18$, no muestra los tabiques de tejido conjuntivo que se introducen en la zona medular (Figura 2), lo que corresponde a una etapa muy temprana del desarrollo gonadal.

En las muestras histológicas a mayor aumento (400x), se observa una gran cantidad de oocitos

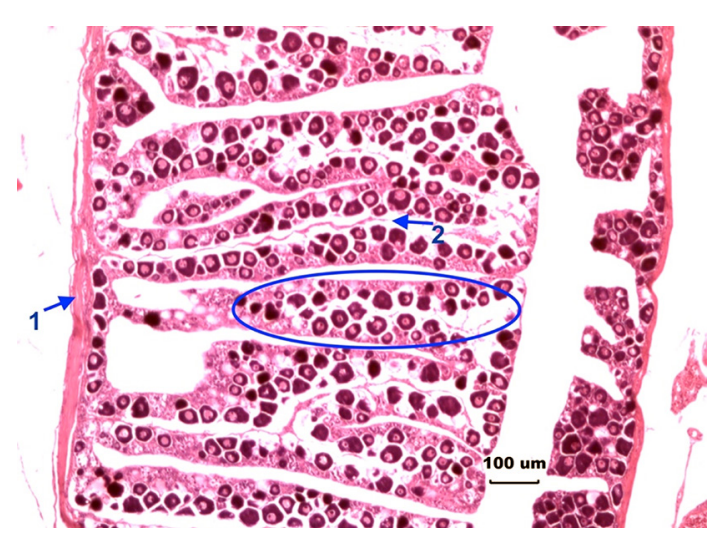

Figura 1. Microfotografía que muestra un corte histológico de una gónada de hembra, correspondiente a una muestra $\mathrm{N}^{\circ} 10$, con un peso de $153,52 \mathrm{~g}$ y $24,5 \mathrm{~cm}$ de longitud, la flechas 1 indica el tejido conjuntivo que rodea completamente a la gónada (albugínea ovárica), luego este tejido se introduce en la zona medular como lo indica la flecha 2, para formar lóbulos, en su interior se observa una gran cantidad de oocitos (elipse) en estado de inmadurez (Hematoxilina - Eosina - 100x). 


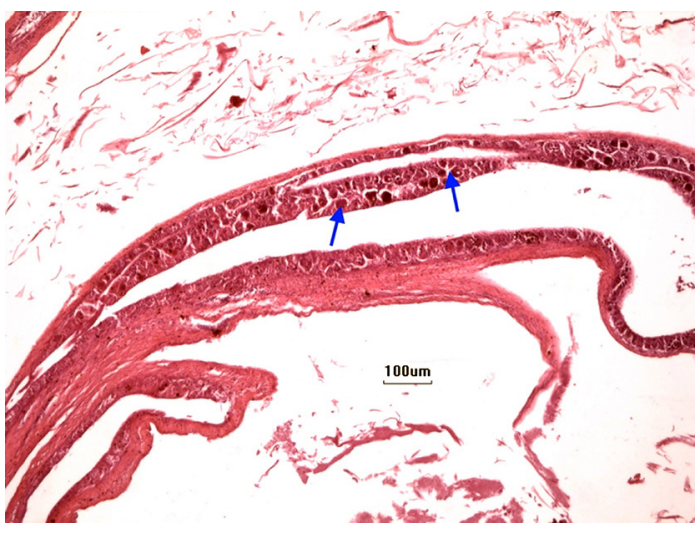

Figura 2. Microfotografía que muestra un corte histológico de una gónada de hembra, correspondiente a la muestra $\mathrm{N}^{\circ} 18$, en donde no se observan los tabiques de tejido conjuntivo propios de la gónada en desarrollo, solo se observan oogonios (flecha) y un conjuntivo muy grueso (Hematoxilina - Eosina - 100x).

en estadios de inmadurez, en donde el núcleo, de características vesiculosas presenta uno o tres nucléolos en su interior adosados a la membrana nuclear (Figura 3), el citoplasma está fuertemente teñido de un azul oscuro y en algunos casos su proporción de volumen, es menor en relación al núcleo.

En la Figura 4, se observa que el tejido gonadal es bastante escaso, en cambio la cantidad de tejido conjuntivo que rodea a los oocitos en maduración, es mayor.

A las muestras que se le aplicó, técnicas de histoquímica, en particular (tinción de Picro-Fucsina

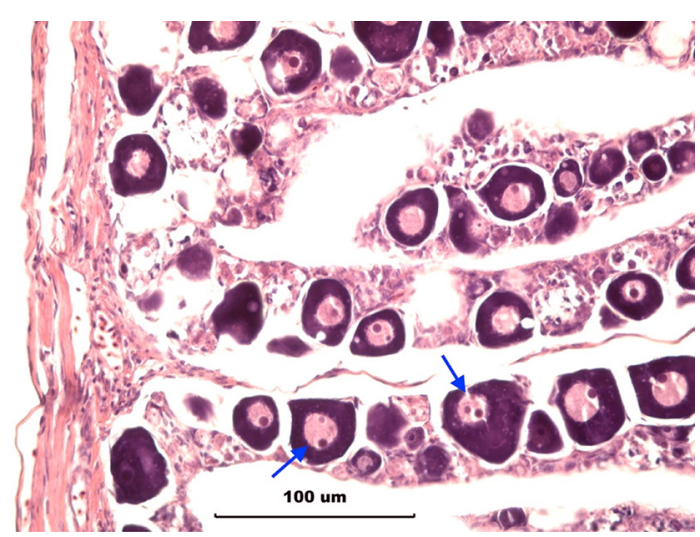

Figura 3. Microfotografía que muestra un corte histológico de una gónada de hembra, correspondiente a la muestra $\mathrm{N}^{\circ} 10$, en donde se observan los oocitos con sus núcleos de características vesiculosas bien definidos, con la presencia de nucléolos (flecha) al interior del núcleo (Hematoxilina - Eosina 400x). de Van Gieson), se identificaron una gran cantidad de fibras colágenas componente típico del tejido conjuntivo denso regular, podemos decir que en el caso de la Figura 5, se observa con claridad una delgada capa de fibras colágenas, las que están involucradas en la formación de la albugínea ovárica durante el desarrollo y maduración de la gónada, además se observa que el mismo tipo de tejido, pero menos consistente que rodea a los oocitos hacia el interior de la medula gonadal. También se pueden observar una serie de vasos sanguíneos, arteriolas y vénulas que están relacionadas con la nutrición y oxigenación del tejido en desarrollo.
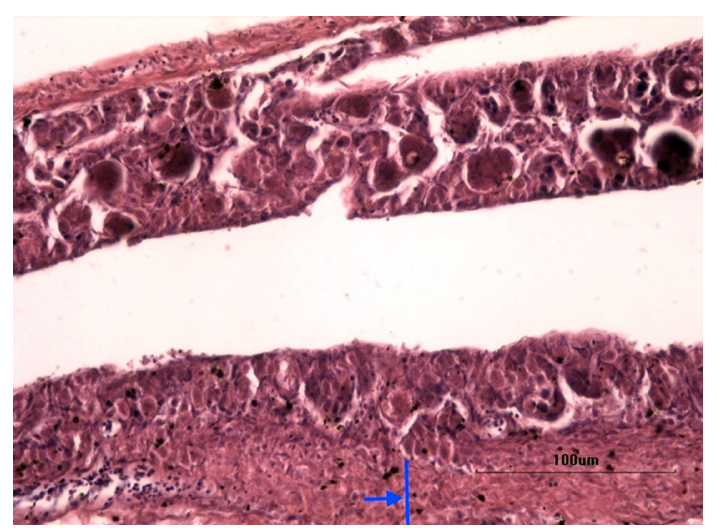

Figura 4. Microfotografía de un corte histológico de la muestra $\mathrm{N}^{\mathrm{o}} 18$, en donde destaca el grosor del tejido conjuntivo (flecha y barra) de la gónada en desarrollo (Hematoxilina - Eosina 400x).

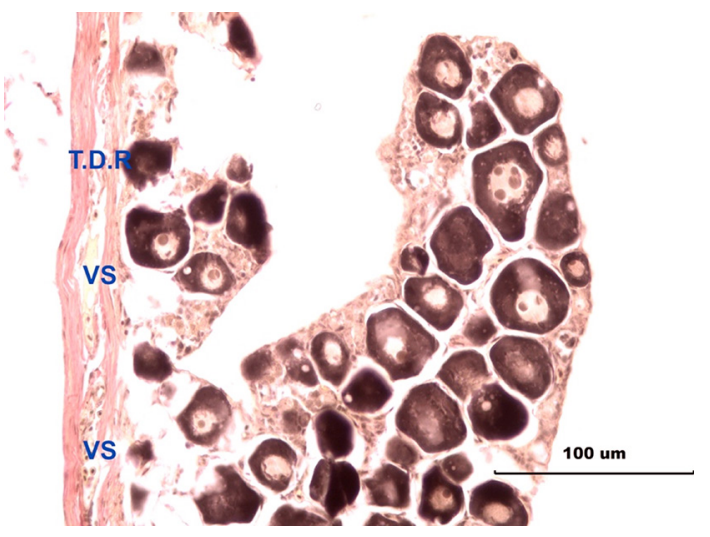

Figura 5. Microfotografía histológica de gónada de Seriola lalandi 400x teñidas con Picro - Fucsina de Van Gieson que corresponde a la muestra $\mathrm{N}^{\mathrm{o}} 10$. Se observan vasos sanguineos (VS) y en su interior glóbulos rojos nucleados, con tejido conjuntivo denso regular (T.D.R), en donde predominan las fibras colágenas. 
En la Figura 6, al comparar con la Figura 5, podemos observar que en general corresponde a una gónada de menor desarrollo por la falta de tabiques de tejido conjuntivo hacia el interior de la gónada, como también una albugínea ovárica mucho más gruesa y con mayor cantidad de vasos sanguíneos.

Al utilizar la técnica histoquímica tintorial del Periodic Acid Schiff (P.A.S.), se observó que el tejido conjuntivo denso regular que rodea a la gónada es P.A.S. negativo, lo que nos indica que en este tipo de tejido no existen hidratos de carbono asociados a proteínas o a otras macromoléculas (mucopolisacáridos o glicoproteínas). Además, los oocitos con aumento de 400x, se observan los núcleos (nucléolos) hay una reacción P.A.S. positiva (Figura 7), en cambio, en la Figura 8, existe una reacción P.A.S. negativa, esto indica la presencia de oogonios de menor tamaño y a la vez muy inmaduros.

\section{Discusión}

Seriola lalandi es una especie seleccionada por el Programa de Diversificación de la Acuicultura Chilena (PDACh), que es coordinada por Innova Corfo y Fondef de Conicyt, con el objetivo de darle un impulso a la acuicultura chilena. En nuestra región de Arica y Parinacota, se proyecta que esta especie iniciará la acuicultura marina y en particular esta investigación es un aporte de gran importancia para poder empezar la reproducción comercial es necesario poder identificar la maduración gonadal .

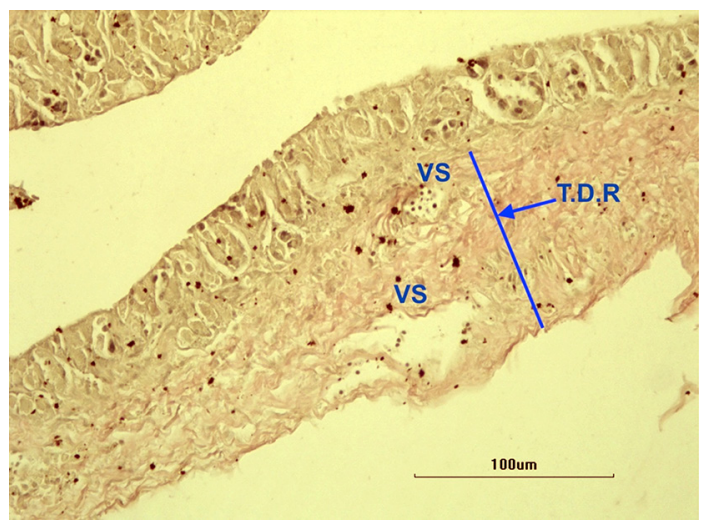

Figura 6. Microfotografía histológica de Seriola lalandi 400x teñidas con Picro - Fucsina de Van Gieson que corresponde a la muestra $\mathrm{N}^{\circ} 18$. Se observa una gran capa de tejido conectivo denso regular teñido de un color más rojizo que el resto del tejido gonadal (T.D.R) y vasos sanguíneos (VS).

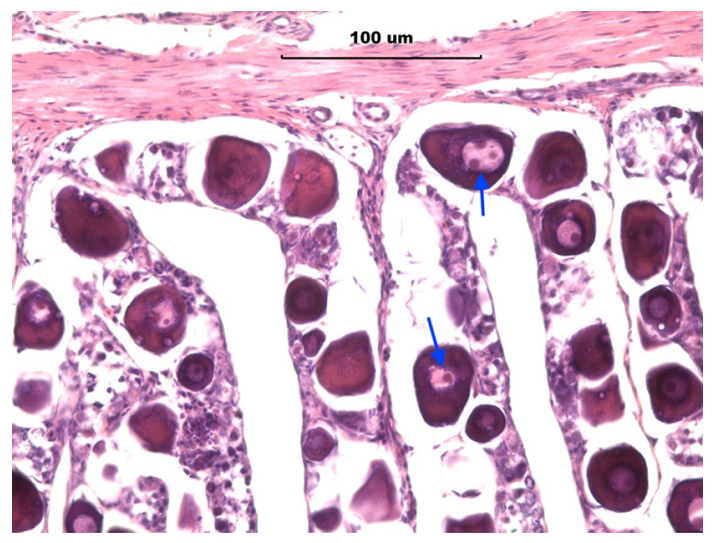

Figura 7. Microfotografía histológica de Seriola lalandi 400x teñidas con P.A.S. que corresponde a la muestra $\mathrm{N}^{\circ} 10$, se observa una reacción P.A.S. positiva (flecha) en el núcleo.

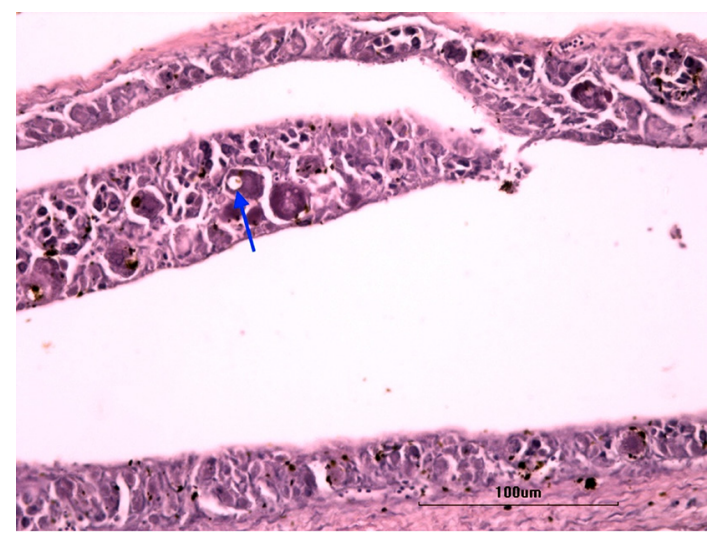

Figura 8. Microfotografía histológica de Seriola lalandi 400x teñidas con P.A.S. que corresponde a la muestra $\mathrm{N}^{\circ} 18$, se observa una reacción P.A.S. negativa (flecha) en el núcleo y se aprecia una cantidad de ovogonios.

Los estudios histológicos basados en los métodos histoquímicos aplicados a las gónadas de Seriola lalandi, como es la técnica de Picro - Fucsina de Van Gieson, mostró que la gónada de las sies hembras, se encuentra recubierta de un tejido albugíneo, con predominancia de fibras colágenas, además, se observa que este tejido conjuntivo está bastante irrigado por abundantes vasos sanguíneos, como lo muestra la Figura 6. Así como lo describen los estudios histológicos realizados por Poortenaar et al. (2001), especialmente respecto de la maduración histológica de la maduración gonadal y no de la morfocitología completa.

El análisis de las gónadas con la tinción del Periodic Acid Schiff (P.A.S), permitió determinar 
que la etapa del desarrollo gónadal de las células sexuales de las hembras, según la tabla de Poortenaar et al. (2001), en los especímenes estudiados se encuentra en estado inmaduro. Es interesante continuar con el análisis de la maduración gonadal a tamaños mayores del pez, y así comprobar si en cautiverio presenta la misma sincronía de desove que reporta Poortenaar et al. (2001), para los peces silvestres capturados en mar abierto. Como también determinar en qué momento de la edad y tamaño del pez, se visualizan claramente las gónadas en la cavidad abdominal.

Las células sexuales de las hembras que se determinaron son los oogonios y oocitos en etapa inmadura, estas células presentaron núcleos y nucléolos muy grandes frente, a un citoplasma muy escaso detallada por Poortenaar et al. (2001), para la determinación de los diferentes estadios de las hembras de Seriola lalandi, además, esta inmadurez está directamente relacionado a los tamaños de los peces que se utilizaron para esta investigación que, en promedio, es de $21,8 \mathrm{~cm}$, siendo muy inferior a los reportados Fielder \& Heasman (2011).

En relación con los resultados obtenidos se realizó el estudio citomorfológico de hembras de Seriola lalandi, ya que las gónadas son de tamaños inferiores a $40 \mathrm{~mm}$, las que se confunden con el intestino que se encuentra en la misma zona anatómica de la gónada, todos los estudios reportados para esta especie trabajan con especímenes de tamaños superiores a los $50 \mathrm{~cm}$ que se alcanza a los dos años o más.

\section{Literatura Citada}

Aguilera, E.; Yany, G.; Romero J.

2013. Cultivable intestinal microbiota of yellowtail juveniles (Seriola lalandi) in an aquaculture system. Latin American Journal of Aquatic Research, 41 (3): 395-403.

Fielder, S.; Heasman, M.

2011. Hatchery Manual for the production of Australian Bass, Mulloway and Yellowtail Kingfish. Publicado por Industry and Investment NSW, $176 \mathrm{p}$.

Martínez-Montaño, E.; González-Álvarez, K.; Lazo, J.; AudeloNaranjo, J.; Vélez-Medel, A.

2014. Morphological development and allometric growth of yellowtail kingfish Seriola lalandi V. larvae under culture conditions. Aquaculture Research, 47 (4), 1277-1287.

Ottolenghi, F.; Silvestri, C.; Giordano, P.; Lovatelli, A.; New, M. 2004. Capture-based Aquaculture: The fattening of Eels, groupers, Tunas and yellowtails. Food and Agriculture Organization of the United Nations, 308 p.
Poortenaar, C.; Hooker, S.; Sharp, N.

2001. Assessment of yellowtail kingfish (Seriola lalandi lalandi) reproductive physiology, as a basis for aquaculture development. Aquaculture, 201: 271-286.

Ruiz, V.H.

2012. Peces: Generalidades sobre su biología y clasificación. En Arana, P.M. (Ed.), Recursos pesqueros del mar de Chile. Escuela de Ciencias del Mar. PUCV. Valparaíso, Chile. pp. 255-286.

Shiraishi, T.; Ohshimo, S.; Yukami, R.

2010. Age, growth and reproductive characteristics of gold striped amberjack 1 Seriola lalandi in the waters off western Kyushu, Japan. New Zealand Journal of Marine and Freshwater Research, 44 (2): 117-127.

Silva, K.

2011. En Chile: estado de potenciales especies para diversificar la acuicultura. Mundo Acuícola Pesquero, 82: 6-8. 
